\title{
Chapinero en la red categorial de los patrones de ocupación del suelo urbano*
}

Fecha de recepción: 11 de abril de 2013 Fecha de aceptación: 13 de marzo de 2014

Juan Manuel Bueno Carvajal

Arquitecto $\quad$ Docente, Universidad Piloto de Colombia

juaneme87@hotmail.com, juan-bueno@unipiloto.edu.co

Resumen El presente artículo aborda el tema de los patrones de ocupación del suelo urbano a partir de sus definiciones desde la gestión y el urbanismo que se agrupan en una red categorial, la cual permite concentrarlos en cinco grandes grupos: suelo, infraestructura, zonificación, construcción e integrales. Para contextualizar dicha red, la investigación aborda un contexto de aproximación en las dimensiones de América Latina, Colombia, Bogotá y, por último, el caso de estudio: la localidad de Chapinero. La red categorial de los patrones es un instrumento de caracterización de la localidad que, como resultado, expone a Chapinero en nueve zonas que se identifican por la particularidad y las dinámicas de los patrones.

Palabras clave Patrones de ocupación; tipología; gestión urbana; Bogotá; localidad; Chapinero

Palabras clave Espacio público; espacio urbano; administración urbana-Chapinero

descriptor (Bogotá, Colombia)

Investigación de tesis de Maestría en Gestión Urbana, perteneciente a la línea de investigación de Hábitat y territorio de la Universidad Piloto de Colombia, iniciada en el año 2011 y finalizada en 2013. 


\section{Chapinero in the Categorial Network of Patterns of Urban Land Occupation}

Abstract This article addresses the issue of urban land occupation patterns from their definitions from the management and planning that are grouped into a categorical network, which allows concentrating them on five major groups: land, infrastructure, zoning, construction and integrals. To contextualize this network, the research addresses the context of approximation in the dimensions of Latin America, Colombia, Bogota, and, finally, the case study: the city locality of Chapinero. The categorical network of patterns is a tool for characterization of the locality, as a result, exposes Chapinero in nine areas identified by the peculiarity and the dynamics of the patterns.

Key words Occupancy patterns; typology; urban management; Bogotá; locality; Chapinero

Key words Public space; urban space; urban management -Chapinero (Bogotá, Colombia) plus

\section{Chapinero na rede categorial dos padrões de ocupação do solo urbano}

Resumo O presente artigo aborda o tema dos padróes de ocupação do solo urbano a partir das definiçôes desde a gestão e o urbanismo que são agrupadas em uma rede categorial, a qual permite concentrá-los em cinco grandes grupos: solo, infraestrutura, bonificação, construção e integrais. Para contextualizar tal rede, a pesquisa aborda um contexto de aproximação nas dimensôes da América Latina, Colômbia, Bogotá e, por fim, o caso de estudo: a localidade de Chapinero. A rede categorial dos padróes é um instrumento de caracterização da localidade que, como resultado, expóe Chapinero em nove zonas identificadas pela particularidade e as dinâmicas dos padróes.

Palavras chave Padrões de ocupação; tipologia, gestão urbana; Bogotá; localidade; Chapinero

Palavras chave Espaço público; espaço urbano; planejamento urbano-Chapinero descritor (Bogotá, Colombia) 


\section{Introducción}

Con el paso de los años, las ciudades en general evidencian transformaciones en los patrones de ocupación del suelo. De estos cambios se destacan tres aspectos: el crecimiento de la población, la expansión urbana y los cambios en la morfología.

Respecto al aumento de la población, se evidencia en América Latina que este ha sido un fenómeno rápido y puede sostenerse que el sello distintivo de la redistribución de la población en el espacio ha sido su acelerada urbanización, es decir, el veloz incremento del porcentaje de habitantes de localidades urbanas (Rodriguez y Villa, 1998). 1950 y $2010^{2}$.
En cuanto a la expansión de la ciudad, Bogotá admite una lógica horizontal que implica una acelerada ocupación del suelo, controlada solo por límites geográficos (Nullvalue, 2009)33. La atracción que provoca la capital radica en su oferta de oportunidades colectivas puesto que no es exclusiva para el ámbito privado y es tan grande como la misma necesidad de suelo para alojar a varios millones de personas (Saldías Barreneche, 2007).

Existen algunos hechos relacionados con modelos de expansión a lo largo de la historia reciente de la ciudad. Se han contemplado modelos europeos de ciudad densa central o norteamericanos de suburbio en la década del sesenta por el Concejo de Bogotá. El economista Launchlin Currie (1988) planteó la dificultad de aplicar el modelo de suburbio norteamericano en países del tercer mundo. Los costos de la ciudad de baja densidad solo pueden ser soportados por una economía sólida y poderosa, capaz de mantener la infraestructura vial. Currie definió, entonces, los dos temas a los que las políticas públicas deberían apuntar: la organización del transporte público y la forma de gestionar el suelo sobre el cual se desarrollará la ciudad.

En relación con la estructura morfológica, Bogotá posee un esquema reticular tradicional que

1 En 1964, Bogotá contaba con 1.861.913 habitantes; Barranquilla con 498.301; Cali con 637.929 y Medellín con 772.887. Hacia 1973, Bogotá registró 286.193 habitantes; Barranquilla, 703.488; Cali, 991.549 y Medellín, 1.163.868. Para 1985, Bogotá alcanzó 4.236.490 habitantes; Barranquilla, 927.233; Cali, 1.429.026 y Medellín, 1.480.382. En 1990, las cuatro ciudades, en el mismo orden, registran $4.716 .801 ; 988.495 ; 1.562 .686$ y 1.553 .648 (DANE, 2005).

2 Desde 1964 y durante nueve años, Bogotá creció dos tercios y se duplicó doce años después. En la década del noventa disminuyó el crecimiento, pues solo aumentó en 500.000 habitantes. Este panorama es similar para las otras ciudades capitales: Cali presentó el crecimiento más acelerado en el mismo lapso y, de la década del sesenta a la del noventa, creció aproximadamente en un millón de habitantes.

3 Al oriente, Bogotá limita con el borde característico de su imagen natural, los cerros Orientales y al occidente con el río Bogotá. Hacia el último punto se encuentra la sabana de Bogotá, área ocupada por suelo rural, industria agrícola y suelo urbano de municipios cercanos. 
ha configurado el orden de la ciudad por medio de algunos ejes viales primarios. La disposición vial impone una tensión en sentido sur-norte que propone un tejido relativamente uniforme y consolidado en esta dirección. Los desarrollos urbanos sin planeación (barrios que, en un principio, fueron ilegales en localidades como Tunjuelito y Engativá) y con planeación (barrios construidos por el Banco Central Hipotecario, Pedro Gómez, Fernando Mazuera, entre otros) aportan a esta configuración morfológica unas piezas que se van integrando y complementan el tejido morfológico mixto que no logra homogenizar alguna identidad y unidad urbana.

$\mathrm{Al}$ momento de referirse a la consolidación urbana, es clave el aspecto morfológico, ya que mediante este una ciudad da cuenta de su forma, composición y las determinantes que han influenciado su constitución. La forma urbana no se compromete con un análisis abstracto o con las justificaciones de distintos comportamientos sociales de la ciudad; es por medio de esta que se entiende cómo y por qué se han configurado las ciudades a partir de la forma como receptáculo de significado (Kostof, 2009).

Como resultado de estas tres transformaciones que afectan la ciudad surge la inquietud de conocer los patrones de ocupación y hacer un ejercicio de categorización a partir de un caso de estudio -la localidad de Chapinero, de Bogotá- y así aportar elementos para caracterizar la zona, con posibilidad de replicarse en otras localidades y quizás en ciudades con una escala similar. La investigación que origina el presente artículo pertenece a la Maestría en Gestión Urbana de la Universidad Piloto de Colombia, donde el tema principal son los patrones de ocupación revisados desde el tema histórico implementado en el caso de estudio.

En este texto el lector podrá conocer las distintas dimensiones abordadas en la indagación, desde las ópticas de los autores que conforman el estado de conocimiento de la investigación: el contexto del caso de estudio a partir de la dimensión de América Latina, Castells (1971) y Gouëset (1998); la dimensión de Colombia, Gouëset (1998) y Janoshka (2006) y la dimensión de Bogotá, Alexiou (2011). Segundo, la puntualización del caso de estudio, la localidad de Chapinero (constitución y datos generales de la periodización correspondiente a la segunda mitad del siglo XX). Tercero, la red categorial de las distintas definiciones y tipologías de los patrones de ocupación y la metodología de categorización para su identificación en la localidad. Por último, las conclusiones de esta experiencia de análisis y la postulación de otras metodologías que complementen la categorización.

\section{Contexto de la investigación}

El entorno relacionado con el estudio de los patrones de ocupación es abordado con un cambio de escala, desde el medio más amplio hasta el más particular. Se parte del contexto de América Latina y se llega a la localidad de Chapinero.

Figura 1.

Contexto: América Latina, Colombia, Bogotá, Chapinero

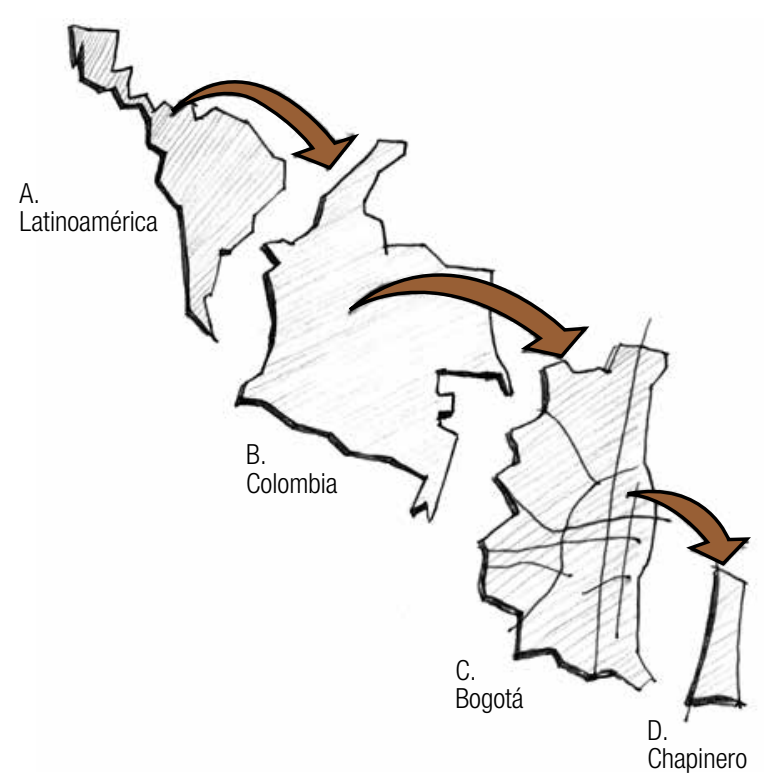

Fuente: elaboración propia 


\section{América Latina}

La semejanza entre varios países de América Latina en los procesos de urbanización se concentra en un crecimiento unipolar, en el que una ciudad tiene la supremacía de crecimiento con respecto a otras (Gouëset, 1998). Se pueden contemplar tres maneras de explicar el carácter primacial de estas redes urbanas:

La primera es la teoría de la urbanización independiente; esta plantea el término de macrocefalia urbana (Castells, 1971) para explicar el carácter único de crecimiento de América Latina, donde se alcanzarán unos índices acelerados de crecimiento y expansión. El término de macrocefalia viene del término médico hidrocefalia; en el caso urbano, esta es una patología de las ciudades latinoamericanas, en la que población y actividades se concentran de manera excesiva en un número reducido de ciudades y ocasionan un obstáculo de desarrollo.
Latina, que fortalece la primacía urbana (Janoshka, 2006).

\section{Colombia y las regiones}

Colombia es una excepción al modelo de primacía urbana, puesto que la concentración urbana se generó en cuatro ciudades principales: Bogotá, Medellín, Cali y Barranquilla. Este auge no se debe solo al tema demográfico. Las cuatro grandes ciudades crecieron por su particular dinamismo en el plano económico, puesto que representan casi la mitad del Producto Interno Bruto (PIB $)^{4}$ de toda la Nación y cada una presenta una economía diferenciada. Bogotá, capital nacional, se aparta de actividades de producción; Medellín es centro cafetero, minero e industrial; Cali se destaca por actividades agroindustriales y Barranquilla es sede de la actividad portuaria.

Figura 2.

Cuadricefalia y las regiones en Colombia

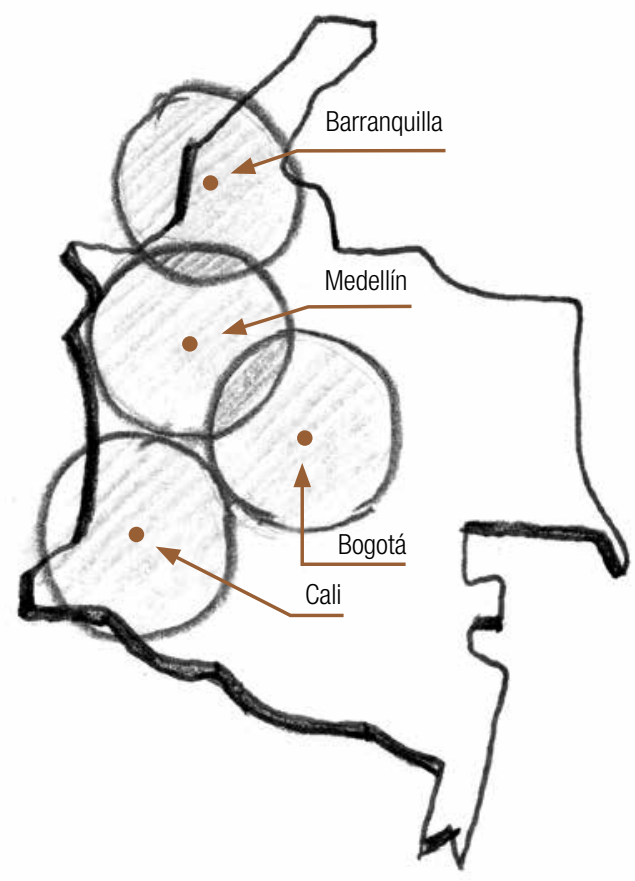

Fuente: elaboración propia

4 Entre 2001 y 2007, el PIB de Colombia mostró una notoria tendencia de crecimiento, con tasas superiores a un 4\% desde 2003. En 2011 se destacaron con mayor PIB per cápita la ciudad de Bogotá y los departamentos de Antioquia, Valle, Santander, Atlántico y Meta y el PIB nacional creció en un 5,9\% (Centro de estudios de la construcción y el desarrollo urbano y regional [Cenac], 2013). 
Para definir el desarrollo urbano del país se asume el término de cuadricefalia (Gouëset, 1998), que expone el esquema dominante de organización del espacio físico del país. Esta organización se puede explicar mediante cuatro argumentos:

- Las limitaciones físicas, sobre todo el relieve, pues las masas montańosas separan los principales núcleos de población.

- El desarrollo incompleto y tardío de la red de vías de comunicación.

- La distribución espacial de la población colombiana antes de la fase de aceleración de urbanización (segunda mitad del siglo XX), pues el país era principalmente rural y la población estaba dispersa.

- El país tiene una estructura económica regional con descentralización de las actividades productivas.

\section{Bogotá}

En el caso de Bogotá existe una complejidad ocupacional y espacial del suelo que puede abordarse a partir de la dimensión social:

[...] la fragmentación y la segregación transformaron el patrón centro/periferia en un patrón norte/sur, es decir, lineal, y esta división social fue acompañada de la consolidación funcional en torno a los principales ejes viales, como eje terciario centro/norte, y los ejes industriales centro/occidente (Alexiou, 2011, p. 27).

Bogotá asumió un esquema de ocupación espontáneo, alejado del control del Estado durante los fenómenos migratorios que consolidaron agrupaciones ilegales y que expandieron zonas de la ciudad de manera libre. Esta incapacidad de control estatal trae graves consecuencias urbanas que afectan hoy la calidad de vida ${ }^{5}$ de los ciudadanos. La ciudad creció de manera desordenada con una tendencia horizontal y sobrepasó cualquier límite físico urbano de control.

\section{Evolución y constitución de la localidad de Chapinero}

La localidad de Chapinero fue escogida a partir del principio de cumplimiento de la diversidad necesaria para identificar los patrones de ocupación del suelo urbano.

Contenido dentro de la temporalidad determinada para el estudio, el término de localidad no aplica sino desde 1991. Por lo anterior, el lapso comprendido desde la década del cincuenta hasta la fecha mencionada tiene otras características

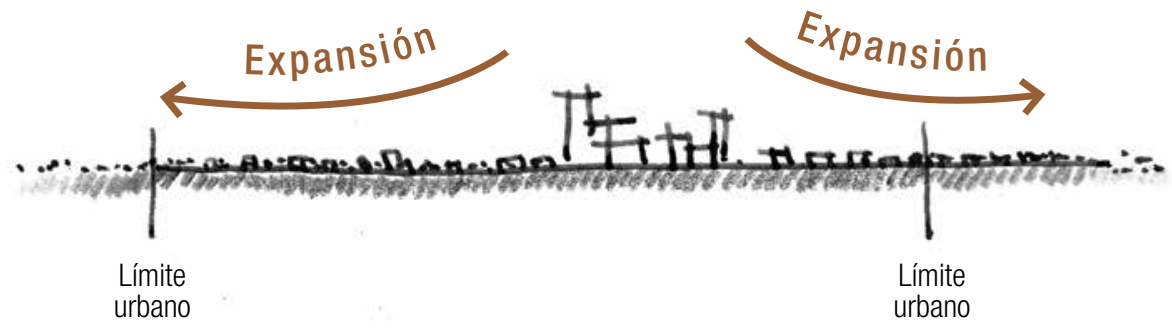

Fuente: elaboración propia 
que vale la pena puntualizar. A continuación se exponen las transformaciones territoriales desde la organización administrativa de la ciudad enmarcado en tres fases históricas: 1954, distrito especial; 1970, Alcaldía menor y 1991, localidad.

\section{4: distrito especial}

Tras El Bogotazo (9 de abril de 1948), en algunos gremios se generalizó la idea de que la tierra se "liberó", lo que sucedió de una forma casi literal y más rápida que la acción de años de leyes urbanas y reglamentos municipales (De Urbina y Zambrano, 2009). Chapinero sufrió cambios importantes en términos administrativos. Previo a esta fecha se denominaba caserío y, debido al crecimiento del barrio Teusaquillo, la clase alta migró hacia Chapinero (de centro a norte). Establecido ahora como zona, pertenece al distrito especial de Bogotá creado por el Decreto 3640 de 1954 y fue gobernado por un alcalde menor.

\section{0: Alcaldía menor}

ciudad que incluye la gestión de recursos propios. Este contexto administrativo está oficializado por la Ley 1421 de 1993 que regula el modelo fiscal, administrativo y político para todas las localidades de Bogotá6. Desde entonces, Chapinero es administrada por siete ediles, las JAL y el alcalde menor.

\section{Chapinero territorial y poblacional}

Geográficamente, la localidad limita al oriente con los cerros orientales y con los municipios de Choachí y La Calera; al occidente con la avenida Caracas y la autopista Norte y las localidades de Teusaquillo y Barrios Unidos; al norte con la calle 100 y la localidad de Usaquén y al sur con el río Arzobispo y la localidad de Santa Fe.

Tiene una extensión de 3.899 hectáreas y una población aproximada de 122.089 personas, lo que equivale a un $1,80 \%$ del total de la ciudad (Secretaria Distrital de Cultura, Recreación y Deportes, 2008). Por el número de habitantes, Chapinero se asemeja a ciudades de Colombia como Duitama (101.210 habitantes), Yopal (117.935 habitantes) y Piedecuesta (115.902 habitantes) (DANE, 2005).

Dentro de los debates de gestión del siglo pasado, se encuentra el de conurbación de los municipios cercanos, los cuales fueron incluidos en Bogotá tras el crecimiento de la ciudad. Para este caso, es importante sostener que la conurbación fue más que un hecho urbano de expansión: Chapinero era una esperanza que repercutió en una visión general de mejora de la ciudad de Bogotá, puesto que sus grandes fincas y hermosos paisajes ofrecieron un estilo y una calidad de vida inimaginables y, al ser incluida en la ciudad, equilibraría al resto de la capital que progresivamente crece bajo un esquema diferente (Cuéllar y Mejía, 2007).

6 Las dieciséis localidades de la ciudad de Bogotá son Antonio Nariño, Mártires, Santa Fe, Fontibón, Barrios Unidos, Bosa, Rafael Uribe, Chapinero, Engativá, Ciudad Bolívar, Kennedy, La Candelaria, Puente Aranda, San Cristóbal, Suba, Sumpaz, Teusaquillo, Usme y Usaquén. 


\section{Caracterización y red categorial de los patrones en Chapinero}

Como proceso metodológico, antes de exponer la red categorial cabe puntualizar en el concepto central de patrones de ocupación. Estos se comprenden como la forma en la que el terreno de una ciudad está dispuesto en lotes o parcelas y se orientan las construcciones. Estas disposiciones tienden a variar, debido a la influencia de transformaciones relacionadas con varias dimensiones de la gestión urbana: usos del suelo en la demanda de una utilización específica, normatividad, renovación, infraestructura nueva y conurbación, entre otros. La forma como se ocupa el suelo de la ciudad está vinculada con temas de composición y función urbana, además de la imagen e identidad de las ciudades.
Ahora bien, de la búsqueda bibliográfica que permitió construir la anterior definición, se resumen veintiún conceptos teóricos de los patrones de ocupación, formulados por seis autores desde 1985 hasta 2010. Cada uno tiene enfoques de distintas posturas, desde la morfología urbana de los franceses Alan Borie y Francois Denieul (1985), las prácticas interdisciplinarias del urbanismo del español Manuel de Solá-Morales (1986), la geografía del español Horacio Capel (2002), la planeación de la colombiana Patricia Rincón (2007), el ordenamiento territorial de la firma española Casariego/Guerra (2008) y la ecología del español Francisco Aguilera (2010). En la Tabla 1 se muestra la base de datos de los patrones de ocupación agrupados por los autores mencionados y dispuestos de manera cronológica.

Tabla 1.

Base de datos patrones de ocupación

\begin{tabular}{|c|c|c|c|c|c|}
\hline \multicolumn{6}{|c|}{ PATRONES DE OCUPACIÓN } \\
\hline \# & Año & Autor & DEFINICIÓN & $\begin{array}{l}\text { TIPOS - } \\
\text { MODALIDADES }\end{array}$ & DESCRIPCIÓN \\
\hline 1 & $\begin{array}{l}\stackrel{\infty}{\infty} \\
\text { வ }\end{array}$ & 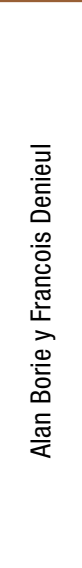 & $\begin{array}{l}\text { Los patrones de ocupación están dentro del } \\
\text { marco de la exigencia de los arquitectos por } \\
\text { reflexionar sobre el patrimonio de la ciudad. Son } \\
\text { una responsabilidad en la conservación y rehabili- } \\
\text { tación urbana a partir de esta visión fisco-morfo- } \\
\text { lógica. La base de la composición de los patrones } \\
\text { es el tejido urbano. La propuesta metodológica } \\
\text { consta de la ampliación de escala, esto permite } \\
\text { hacer una descomposición del tejido urbano en } \\
\text { cuatro subsistemas: los lotes o predios, las vías, } \\
\text { las construcciones y los espacios libres. Esta sub- } \\
\text { división no es estática y hace ver la relación entre } \\
\text { cada uno de estos componentes, esta puede ser } \\
\text { de interdependencias, dependencias, superposi- } \\
\text { ciones, aislamientos, etc (Borie y Denieul, 1985). }\end{array}$ & $\begin{array}{l}\text { Lotes } \\
\text { Vías }\end{array}$ & $\begin{array}{l}\text { Este sistema consta de una repartición del espacio } \\
\text { del territorio en un número de unidades funcionales, } \\
\text { que son los lotes, sinónimos de predios o parcelas. } \\
\text { Es el sistema de unión del espacio del territorio, se } \\
\text { articula por medio de las circulaciones que tienen } \\
\text { como propósito comunicar a los lotes. } \\
\text { Son el conjunto de volumetrías de la forma urbana. } \\
\text { No interesa su uso, función o dimensión. Pueden } \\
\text { ser puntuales, lineales o plenas. } \\
\text { Son las partes no construidas del tejido urbano. } \\
\text { Pueden ser privadas o públicas. }\end{array}$ \\
\hline 2 & 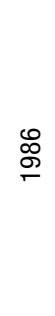 & 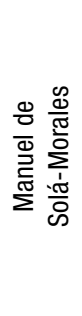 & $\begin{array}{l}\text { Desde la geografía, se refiere a los patrones de } \\
\text { ocupación desde una visión morfológica, que se } \\
\text { relaciona con las diferentes tipologías urbanas. }\end{array}$ & $\begin{array}{c}\text { Las tipologías } \\
\text { estructurantes } \\
\text { del crecimiento } \\
\text { urbano }\end{array}$ & $\begin{array}{l}\text { Estas son el conjunto de operaciones materiales } \\
\text { de construcción de la ciudad según el orden e } \\
\text { importancia de las operaciones: parcelación, urba- } \\
\text { nización y edificación. Es importante conocer que } \\
\text { esta deducción estructural se soporta en una visión } \\
\text { tipológica descriptiva que tiene un fuerte carácter } \\
\text { histórico ya que se obtiene del estudio histórico de } \\
\text { la ciudad por partes y la exigencia de relacionarlo } \\
\text { con sus formas de gestión. }\end{array}$ \\
\hline
\end{tabular}


PATRONES DE OCUPACIÓN

\# Año Autor DEFINICIÓN
TIPOS MODALIDADES

\begin{tabular}{|c|c|}
\hline $\begin{array}{c}\text { Cascos } \\
\text { antiguos }\end{array}$ & $\begin{array}{l}\text { Resultado de la evolución histórica que implementa } \\
\text { matices importantes, el crecimiento de las ciudades } \\
\text { ocurre en lo que se considera el casco antiguo. }\end{array}$ \\
\hline Ensanches & $\begin{array}{c}\text { Van mas allá del concepto de ampliación, implican } \\
\text { una noción de intervención y complejas operaciones } \\
\text { de reparcelación del espacio existente. }\end{array}$ \\
\hline
\end{tabular}

Se refiere a la parcelación de suelo rústico, este se transforma en urbano y aumenta su valor. Interviene

Parcelaciones la construcción de algunas estructuras como vías y privadas redes. Tras esta estructura vial se dividen y definen las parcelas. la investigación de la ciudad, que es un obje Define cinco tipologías de ocupación.

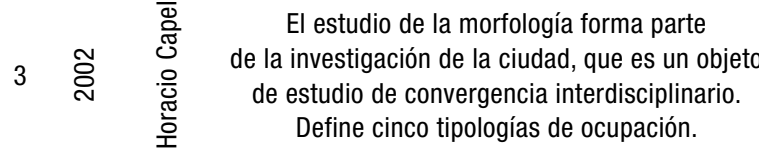

Patrones entendidos como modalidades, las cuales "han sido más características de una época, otras de una tipología de vivienda, o del efecto de normas que ha dictado la 'planificación' de la ciudad, o de cambios en el mercado del suelo 0 de las políticas urbanas de impulso a la construcción, o de un efecto combinado de varias de ellas" (Rincón, 2007, p. 86).

Autocons-
trucción
Tejidos de
ciudad jardín

Se relaciona al problema de vivienda de la ciudad. Cuando la población crece mas rápido que los alojamientos. Esta es una morfología específica en la cual la desorganización predomina.

Son las parcelas ubicadas en espacios no edificados donde su morfología depende del trazado vial, donde las formas orgánicas predominan así como el ajuste a la topografía ocupada.

Substitución individual de edificaciones

Subdivisión
predial en lotes
pequeños

Consiste en la demolición individual de viviendas, preferiblemente unifamiliares, para remplazarlas por edificios de mayor altura, presión del mercado y cambios de norma.

La característica principal de este patrón de ocupación es el tamaño de los predios, el cual varía de acuerdo con los ingresos de los sectores. Son más amplios para los de mayores ingresos y más pequeños para los de menores.

\begin{tabular}{cc}
\hline $\begin{array}{c}\text { Consolidación } \\
\text { de barrios } \\
\text { de desarrollo } \\
\text { progresivo }\end{array}$ & $\begin{array}{c}\text { Esta ocupación se refiere a la densificación a partir } \\
\text { de la ampliación del módulo original inicial. }\end{array}$ \\
\hline $\begin{array}{c}\text { Abandono } \\
\text { y posterior } \\
\text { subdivisión de } \\
\text { viviendas }\end{array}$ & $\begin{array}{c}\text { Edificaciones que han sido abandonadas por sus } \\
\text { dueños, lo cual genera el deterioro del área } \\
\text { y la conformación de inquilinatos. }\end{array}$ \\
\hline $\begin{array}{c}\text { Llenado y } \\
\text { construcción } \\
\text { de lotes vacíos }\end{array}$ & $\begin{array}{c}\text { Consiste en el llenado y ocupación del perímetro } \\
\text { conurbación, es el recurso último de expansión en } \\
\text { el suelo urbanizable de la ciudad. }\end{array}$ \\
\hline $\begin{array}{c}\text { Programas de } \\
\text { renovación } \\
\text { urbana }\end{array}$ & $\begin{array}{c}\text { Para este caso, es claro que generalmente el Estado } \\
\text { adquiere y demuele franjas o manzanas completas } \\
\text { para realizar un proyecto nuevo. }\end{array}$ \\
\hline
\end{tabular}

Hay un cambio de uso y se reutilizan las edificaciones que ya existen para darle un nuevo uso original. Estas sufren considerables modificaciones que en la mayoría de casos son al interior de la edificación. 
PATRONES DE OCUPACIÓN

\begin{tabular}{|c|c|c|c|}
\hline \# & Año & Autor & DEFINICIÓN \\
\hline 5 & 怘 & 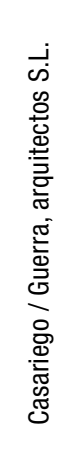 & $\begin{array}{l}\text { Se abordan los patrones por medio de la formula- } \\
\text { ción del Plan Territorial Parcial de Ordenación de } \\
\text { la Comarca de Abonala. Son la forma de ocupar el } \\
\text { suelo, son una determinante o el parámetro más } \\
\text { relevante para entender el consumo del suelo } \\
\text { desde algunas décadas. Son estas tipologías } \\
\text { de asentamiento que se generan por distintos } \\
\text { factores como su uso, la trama urbana y la edifi- } \\
\text { cabilidad. Para la propuesta de revisión de patrón } \\
\text { de ocupación de casariego / Guerra es importante } \\
\text { resaltar el alto componente de determinante fun- } \\
\text { cional para la identificación de la particularidad } \\
\text { de la ocupación. Es una visión netamente de uso. }\end{array}$ \\
\hline
\end{tabular}

TIPOS -

MODALIDADES

DESCRIPCIÓN

\begin{tabular}{cc}
$\begin{array}{c}\text { Suelo } \\
\text { residencial }\end{array}$ & $\begin{array}{c}\text { Divisiones que dependen de los parámetros de } \\
\text { carácter espacial: dependencia con el resto del } \\
\text { sistema comarcal y temporal. Asentamientos } \\
\text { originales en zonas de agricultura. }\end{array}$ \\
\hline Suelo turístico & $\begin{array}{c}\text { Según el tipo de oferta que ocurre en la zona, la } \\
\text { ocupación de este tipo de suelo se puede identificar. }\end{array}$
\end{tabular}

Se define como aquel que cumple con funciones
de mejora de las condiciones de la comunidad en
la que se inserta y se consideran como funciones
complementarias, de apoyo y de contribución
al desarrollo en general.

Las métricas de análisis espacial, son primero, el número de teselas, es decir, la unidad de medida de división o fragmentación. Segundo, el tamaño medio de teselas, correspondiente a la superficie que ocupan las teselas, aporta información sobre las posibles fragmentaciones. Tercero, la compacidad de fragmentos, que corresponde a la distancia entre fragmentos, cuanto más cercanos mayor será su compacidad. Cuarto, el índice de forma, que mide la geometría de los fragmentos con base en su perímetro. Quinto, la distancia media, que es la distancia entre teselas del mismo uso. Sexto y último, el índice de proximidad media, que es la sumatoria de área en $\mathrm{m}^{2}$ de las teselas del mismo uso.

Tras este rastreo bibliográfico se agruparon las nociones en conjuntos, con base en un esquema resultante del mismo ejercicio, luego de extraer las características compartidas entre los patrones de ocupación de los referentes; así, se conformó un esquema de capas (layers) (Figura 4) que formula cinco grandes conjuntos: suelo, infraestructura, zonificación, construcción e integrales.

La defragmentación de las capas del tejido urbano es realizada mediante un esquema de composición urbano, que es abstraído de la base de datos que constituye la lectura.

\section{Suelo}

Es de los patrones, la primera capa del esquema de ocupación y se identifica por ser una base, ya que tiene la capacidad de condicionar y estructurar la disposición de los demás patrones en
Figura 4.

Esquema de capas de ocupación y definición de los cinco conjuntos de patrones de ocupación

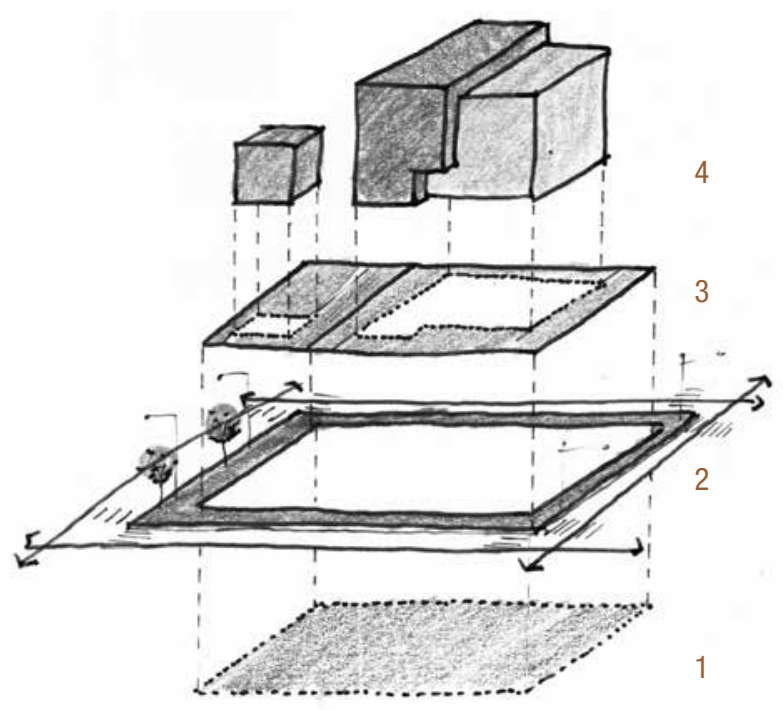

Fuente: elaboración propia 
la ciudad. Se asimila con la capa inicial donde tiene acción la implantación. Se entiende como la subdivisión espacial de las manzanas que conforman la ciudad y son producto de distintas dimensiones de gestión: de las intervenciones de planeación, de reubicación y de restructuración del suelo por implementación de infraestructuras, entre otras.

A este grupo pertenecen los patrones: lotes, ensanches, parcelaciones privadas, tejidos de ciudad jardín, subdivisión predial en lotes pequeños y consolidación de barrios de desarrollo progresivo.

\section{Infraestructura}

Del esquema de ocupación de los patrones, es la segunda capa. Se caracteriza por tener una connotación de direccionamiento del espacio, puesto que lo conforman patrones de vías y de espacio libre que permiten la movilidad en la ciudad.

El gran patrón que predomina dentro de esta agrupación es el del espacio libre, ya que, además de contener al otro patrón (las vías), se rige por un esquema normativo muy claro de ocupación, relacionado con el concepto de índice de ocupación (IO: máxima ocupación de una construcción en un lote) y se presenta en diferentes manifestaciones espaciales que se admiten en dimensiones públicas y privadas como plazas, parques, plazoletas, patios, cesiones, retrocesos, antejardines, alamedas, etc. Los espacios libres contienen la red de espacio público, la cual permite la apropiación y las manifestaciones de los ciudadanos; esto implica otros ámbitos, también de la gestión urbana.

\section{Zonificación}

La tercera capa del esquema ocupacional de los patrones se caracteriza por abarcar los patrones de ocupación que no son físicos. Son una determinante en la identificación de morfologías y partes del siguiente grupo, pero su estructura se relaciona de manera más estrecha con aspectos de la gestión urbana, porque son resultado de mecanismos que definen la posibilidad de caracterizar la estructura de actividades de una ciudad (usos del suelo). Estos medios son gestionados desde planes de renovación, planes de desarrollo y planes de ordenamiento territorial en el ámbito local y permiten zonificar, distribuir y agrupar los usos urbanos.

A esta categoría pertenecen los patrones de programas de renovación urbana, programas de reciclaje, suelo residencial, suelo turístico y suelo logístico. Los tres patrones mencionados se entienden como resultado de un plan parcial de ordenamiento territorial que, para el caso en estudio, pueden ser los usos del suelo local como residencial, comercial, industrial, mixto, institucional, oficinas, etc.

\section{Construcción}

Del esquema de ocupación de los patrones son la cuarta y última capa de lectura, que se define por su condición de visibilidad y reconocimiento. Son volúmenes construidos dentro del espacio urbano. También se entienden como el efecto de los anteriores tres grupos que, por medio de procesos de gestión y planeación, configuraron el suelo para posibilitar su existencia.

Forman parte de esta categoría los patrones de cascos antiguos, autoconstrucción, substitución individual de edificaciones y abandono, construcciones y posterior subdivisión de viviendas. Este grupo se particulariza por tipologías de construcción que siguen las anteriores capas del esquema de patrones.

\section{Integrales}

Este grupo se puede concebir como consecuencia de una metodología de gestión que consta 
de varios procesos o pasos históricos para poder ser identificados. Los integrales no conforman el esquema de capas. Son aquellos que no clasifican en los anteriores grupos, puesto que asumen las cuatro categorías y se posicionan en la ciudad como un patrón que integra varios factores.

Para aclarar esta metodología, es importante puntualizar en los patrones que componen este grupo. El caso del patrón de llenado y construcción de lotes vacíos forma parte de un sistema de gestión de integración urbana conocido como conurbación, la cual absorbe poblaciones cercanas a una ciudad que, debido a su dependencia y a los sistemas creados, generan una expansión que termina en la vinculación de ambos.

En el patrón tipologías estructuradoras del crecimiento urbano se identifican las tres operaciones estructuradoras que articulan esquemas de los primeros cuatro conjuntos: parcelación, urbanización y edificaciones.

Por último, el patrón de métricas de análisis espacial es una metodología de medición del suelo urbano que toma como base una unidad denominada teselas, entendida como una determinada medida de lotes o manzanas que constituyen un esquema integral de ocupación urbana.

Tabla 2.

Red categorial de los patrones de ocupación

\section{CATEGORIZACIÓN DE LOS PATRONES DE OCUPACIÓN}

\begin{tabular}{|c|c|c|c|}
\hline$\#$ & CATEGORÍA & DEFINICIÓN & PATRONES QUE PERTENECEN \\
\hline \multirow{6}{*}{1} & \multirow{6}{*}{ Suelo } & \multirow{6}{*}{$\begin{array}{l}\text { Tienen la capacidad de estructurar y condicionar la disposición } \\
\text { de los demás patrones en el espacio urbano. Se refieren a la } \\
\text { capa inicial donde la implantación tiene acción en la ciudad. }\end{array}$} & Lotes \\
\hline & & & Ensanches \\
\hline & & & Parcelaciones privadas \\
\hline & & & Tejidos de ciudad jardín \\
\hline & & & Subdivisión predial en lotes pequeños \\
\hline & & & Consolidación de barrios de desarrollo progresivo \\
\hline \multirow{2}{*}{2} & \multirow{2}{*}{ Infraestructura } & \multirow{2}{*}{$\begin{array}{l}\text { Posee connotación de direccionamiento en el espacio, ya que } \\
\text { lo conforman los patrones de vías y de espacio libre, los cuales } \\
\text { permiten la movilidad dentro de la ciudad. }\end{array}$} & Vías \\
\hline & & & Espacios libres \\
\hline \multirow{5}{*}{3} & \multirow{5}{*}{ Zonificación } & \multirow{5}{*}{$\begin{array}{l}\text { Su estructura no es física y se relaciona de manera más fuerte } \\
\text { con aspectos de la gestión urbana, ya que son patrones resulta- } \\
\text { do de mecanismos que determinan la posibilidad de caracterizar } \\
\text { la estructura de actividades de una ciudad (usos del suelo). }\end{array}$} & Programas de renovación urbana \\
\hline & & & Programas de reciclaje \\
\hline & & & Suelo residencial \\
\hline & & & Suelo turístico \\
\hline & & & Suelo logístico \\
\hline \multirow{5}{*}{4} & \multirow{5}{*}{ Construcciones } & \multirow{5}{*}{$\begin{array}{l}\text { Se define por su condición de visibilidad y reconocimiento } \\
\text { por ser volúmenes dentro del espacio urbano. }\end{array}$} & Construcciones \\
\hline & & & Cascos antiguos \\
\hline & & & Autoconstrucción \\
\hline & & & Substitución individual de edificaciones \\
\hline & & & Abandono y posterior subdivisión de viviendas \\
\hline \multirow[b]{2}{*}{5} & \multirow[b]{2}{*}{ Integral } & \multirow{2}{*}{$\begin{array}{l}\text { Son aquellos que no clasifican en los anteriores grupos, puesto } \\
\text { que asumen las cuatro categorías y se posicionan en la ciudad } \\
\text { como un patrón que logra integrar varios factores. }\end{array}$} & Llenado y construcción de lotes vacíos \\
\hline & & & Las métricas de análisis espacial \\
\hline
\end{tabular}


Bajo estas cinco categorías se identificaron zonas de análisis con la ayuda de dos fuentes planimétricas, el plano catastral de la localidad de Chapinero (Secretaría Distrital de Movilidad, 2013) y las imágenes satelitales de la localidad, tomadas con el software Google Earth. La primera fuente es oficial y respalda la confiabilidad, veracidad y vigencia de la información y la otra es una fuente no oficial de fácil acceso y manipulación.

\section{Identificación de la red categorial en Chapinero}

El parámetro metodológico para particularizar la zona en estudio es la identificación de la red categorial. Desde el punto de vista teórico, sin contrastar con la realidad, se hace una aproximación a los patrones de los predios catastrales de la localidad, según la categorización de los mismos. Este paso es complementado con la visita en campo que cubrió casi un 70\% de la localidad, cuyo criterio de identificación permitió corroborar los patrones y el acercamiento a zonas no contempladas.

La identificación se realiza con el software AutoCAD mediante las cinco capas correspondientes a las categorías de los patrones, reconocidas con colores diferentes (suelo: verde; infraestructura: naranja; zonificación: rojo; construcciones: amarillo; integral: magenta) y se aplican en cada uno de los predios.

El resultado de este ejercicio se plasmó en el Plano 1, donde se superponen el plano catastral y la imagen satelital, para evidenciar a la localidad inmersa en la ciudad de Bogotá y dar cuenta de su contexto; resaltan elementos urbanos circundantes relevantes como los cerros orientales y otros cuerpos verdes como el complejo del parque Simón Bolívar, al occidente.

\section{Zonificación}

La identificación de los patrones permite zonificar a la localidad, puesto que esta tipificación expone algunas tendencias que señalan particularidades al interior de Chapinero. El Plano 1 revela nueve zonas, que se identificaron bajo un criterio de morfología y de agrupación, evidente por la relación de los colores que representan cada categoría de patrones, coincidentes, en cierto punto, con la relación de barrios catastrales y algún hito urbano ${ }^{7}$ relacionado con estos.

\section{Zona 1: Chicó Norte}

En esta zona se identifica una concentración de patrones pertenecientes a la categoría Construcción, en especial por el tipo de esquema de substitución individual de edificaciones; este reemplazó varias unidades unifamiliares por edificios de alta densidad en suelo urbano de estratos altos. Estos patrones consolidaron, además del uso residencial, el uso de oficinas con presencia en la calle 100, eje lineal sobre el límite norte de la zona y de la localidad. La presión y los cambios normativos permitieron estas transformaciones que casi culminan en 2013 y dejaron apenas algunos rasgos de estándares pertenecientes a la categoría Zonificación, asimiladas a las construcciones que aún conservan su uso y morfología original en residencias unifamiliares o que se relacionan con el esquema de programas de reciclaje donde se conserva la estructura original, pero con un uso nuevo: de residencial a comercial u oficinas. También se detectan concentraciones lineales de modelos de infraestructura, correspondientes a parques vecinales y a importantes ejes viales como la carrera11, carrera 15 y calle 100 , ya mencionada. 
Plano 1.

Categorización de los patrones de ocupación en la localidad de Chapinero

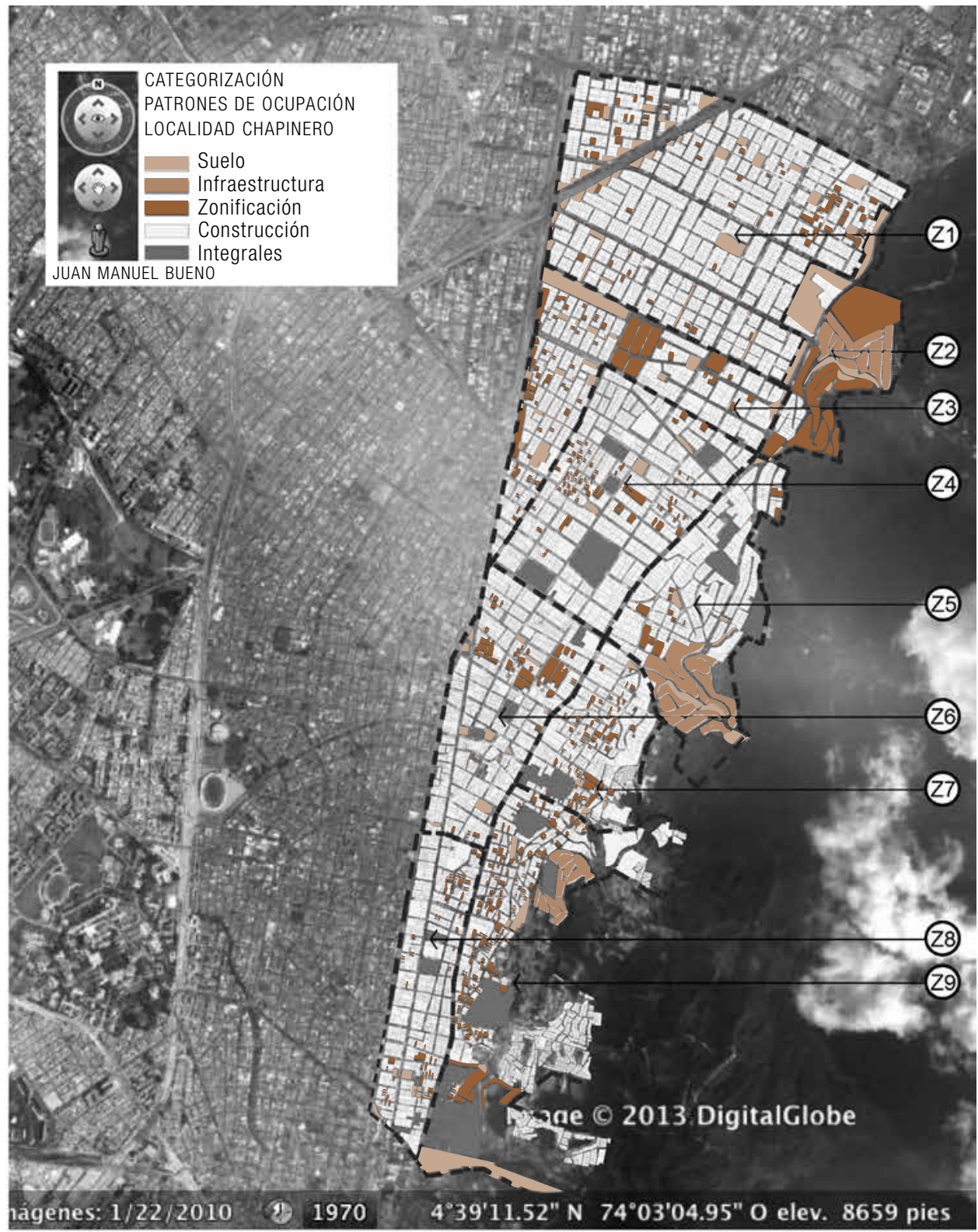

Fuente: elaboración propia con datos tomados de Google Earth (2013) y Secretaría Distrital de Movilidad (2013)

\section{Zona 2: Seminario}

En la categoría Zonificación se halla la principal concentración de patrones en esta zona. Se identifican el icónico edificio del Seminario Mayor de Bogotá, en el cual se reconoce también el esquema de construcción tras la ampliación de la tercera planta de las dos alas del edificio. De la categoría Construcción son, además, edificaciones de vivienda multifamiliar sobre la carrera Séptima y la calle 92, a excepción de la sede administrativa de Bavaria, con uso de oficinas. Este modelo comienza su desarrollo a finales de la década del ochenta y comprende edificaciones de alta densidad en altura. 


\section{Zona 3: Virrey}

En esta área se detecta la concentración de patrones de la categoría Construcción con similares características identificadas en la zona uno. Predomina una gran franja de patrones de infraestructura, que corresponden al parque El Virrey, que a su vez preserva algunos patrones de zonificación pertenecientes a unidades de vivienda unifamiliar ubicados a ambos costados del parque.

En el centro de la zona se identifica un importante elemento articulador de la categoría Zonificación, el cual pertenece al plan parcial de renovación urbana Proscenio, que aún no se ha ejecutado, pero tiene fuerte influencia como eje articulador para esta zona junto al borde occidental, correspondiente a la calle 85 y que pretende ser un hito de ocupación para esta parte de la localidad. El patrón de substitución individual de edificaciones es aquel que modifica gran parte de la zona; se transforma el uso de residencial tradicional, a oficinas y educativo.

\section{Zona 4: Country}

En esta zona se tiende a preservar usos originales con la categorización de Zonificación hacia su límite occidental, junto al eje vial correspondiente a la autopista Norte. De manera particular, en esta área aparece la categoría de patrones integrales, que se identifican en predios correspondientes a equipamientos especiales tradicionales como el Colegio Gimnasio Moderno y la Universidad Pedagógica Nacional. La categoría Construcción se aprecia en algunas transformaciones de edificaciones, donde el suelo cambia de uso y se reconocen nuevos hitos urbanos como los centros comerciales Centro Andino y El Retiro, instituciones educativas técnicas y servicios de salud, entre los que sobresale la clínica del Country.

Se resaltan patrones como el de vías en la tradicional avenida Chile, eje estructurador que desarrolló una de las zonas empresariales y financieras de Bogotá. También el patrón de substitución individual de edificaciones predomina desde la década del ochenta y, por este, el sector se consolidó por completo con nuevos perfiles de edificios en altura con estos usos urbanos relacionados con el comercio y las oficinas.

\section{Zona 5: Rosales}

En esta zona predomina la determinante topográfica que demarca una alta concentración de patrones de la categoría Suelo con tejidos de ciudad jardín, por su morfología no reticular debido a las curvas de nivel, que corresponden a edificaciones de alta densidad de vivienda a lo largo del borde de la quebrada La Vieja. También se identifica el patrón de construcción con edificaciones en altura que transformaron el perfil de viviendas de dos y tres niveles con nuevas estructuras que buscan densificación en altura mediante vivienda multifamiliar. Se reconocen algunos patrones de infraestructura e integrales como el Instituto Colsubsidio de Educación Femenina (CEF) y el Club El Nogal.

\section{Zona 6: Lourdes}

Se identifican de manera puntual y relevante patrones de la categoría Integral y de Infraestructura correspondiente a la iglesia de Nuestra Señora de Lourdes. Junto a este se identifica el patrón de espacios libres en la actual plaza de Lourdes que, tras la última remodelación en 2001, se transformó en espacio duro adoquinado y dejó de ser un parque de manera definitiva, pues conservó poco de su arborización original. Y junto al parque de la 60 (parque de Los Hippies) son íconos en la historia de la localidad.

Se evidencian patrones de zonificación que corresponden a viviendas unifamiliares patrimoniales que se han preservado, al igual que las viviendas multifamiliares. El límite norte de esta zona es un eje vial importante: la avenida Chile, en cuyo costado sur se pueden identificar, en la 
categoría Construcción, patrones en los que todos corresponden a edificios de alta densidad de usos de oficinas como la Bolsa de Valores.

\section{Zona 7: Chapinero Alto}

En esta zona se identifican patrones de la categoría Construcción, pertenecientes a nuevas viviendas en altura y transformaciones de usos de vivienda a comercio en toda la zona. Se destacan patrones integrales como el Club de Oficiales de la FAC y, de manera particular, en el costado oriental junto a los cerros, se presentan en la categoría Construcción los patrones de autoconstrucción por ocupaciones que iniciaron de manera ilegal, como el barrio Bosque Calderón Tejada y Juan XXIII.

\section{Zona 8: Marly}

De manera puntual, en la zona se identifican dos representantes de patrones integrales, que son el Claustro de la Universidad Santo Tomás y la Clínica Marly. Hay una alta concentración de patrones de Construcción que, a diferencia de las demás zonas, en estos no predomina la vivienda en altura, sino el uso de educación en varias construcciones pertenecientes a universidades y equipamientos educativos.

\section{Zona 9: Javeriana}

En la última zona sobresale la categoría Infraestructura, que corresponde al río Arzobispo y el borde norte del parque Nacional. De la misma manera, prevalece la categoría Integral en el campus de la Pontificia Universidad Javeriana, que configuró la categoría de Zonificación con viviendas de densidad media. Se destacan, también en la categoría Integral, las universidades La Salle y Manuela Beltrán y el Hospital Militar. En una concentración resaltan los tejidos de ciudad jardín que poseen una morfología orgánica debido a la topografía y la categoría Suelo, se evidencia en el borde oriental en los cerros, que corresponde al barrio El Castillo.
Del área restante se halla la categoría Construcción en patrones de autoconstrucción con algunos barrios de origen subnormal y algunos patrones de zonificación con viviendas de estilo arquitectónico moderno que aún se conservan.

\section{Conclusiones: aportes del escenario}

En el caso de América Latina, la supremacía urbana es un factor que incide en el conocimiento de los patrones desde un punto de vista de dinámicas de transformación, según la consolidación del escenario urbano. A escala macro, estos patrones revelan que una ciudad dominante y representativa es más propensa a sufrir transformaciones en menor tiempo y, a su vez, que diversificar las características de la forma como se ocupa la ciudad, probablemente influida por cambios de usos al especificarse una función primaria que la destaque. Desde la propuesta metodológica de la red categorial, se pueden esbozar elementos clave en la caracterización de la localidad. De estos se resaltan los siguientes:

\section{Bordes}

Estos se evidencian en la zonificación. En el análisis gráfico es relevante que las agrupaciones de patrones seńalan una delimitación específica dentro de la localidad. Se destacan ejes viales internos que funcionan como límites en el sentido oriente-occidente: la calle 100, la calle 82, la avenida Chile, la calle 57 y la calle 39. En sentido norte- sur: la avenida Carcas, la carrera Séptima y el único límite natural: los cerros Orientales.

\section{Ambiente}

El patrón que puede relacionarse con este aspecto es el de tejidos de ciudad, por su emplazamiento en los cerros Orientales y que debe su morfología a las curvas de nivel. La estrategia de la ciudad para afrontar el ecosistema en donde está implantada —el de esta gran sabana y, en el caso de la localidad, en la falda de los cerros- es 
negligente, ingenua e inconsciente, al no mostrar alguna valoración o relación más allá del aspecto morfológico. Los patrones de ocupación no dieron lección al respecto, pues no estaban orientados a variantes de la ciudad que no contemplan el ambiente.

\section{Cambios de uso del suelo}

Los patrones de ocupación, los programas de reciclaje y la sustitución individual de edificaciones son los principales estructuradores que posibilitaron abordar y afrontar los cambios de usos que tuvo la localidad, tras los que aparecen nuevas dinámicas y concentraciones que transformaron la ocupación y son evidenciados en varios sectores consolidados: la zona 4 Country y la zona 1 Chicó Norte.

\section{Legislación urbana y Administración}

Un factor decisivo en la transformación de la ocupación del suelo de Chapinero es la legislación urbana, que controla distintos aspectos urbanos referidos a crecimiento, desarrollo y consolidación. Dentro de este factor, se halla el papel del Gobierno local y nacional por medio de los mandatarios que inciden en la concepción de la legislación urbana como los planes de desarrollo, estatutos, organizaciones estatales, planes, programas, leyes, decretos, etc. Este ejercicio abre la posibilidad de identificar los hitos normativos que repercuten en los patrones mediante una metodología histórica.

\section{Percepción}

Este aspecto está relacionado con elementos clave dentro de la caracterización de Chapinero como complemento, por medio de otras variables metodológicas que recolecten experiencias de los ciudadanos. Este trabajo deja campo a la intervención interdisciplinar para un análisis urbano que apunte, como resultado, a establecer recorridos urbanos, memorias y mapas mentales.
Desde la propuesta metodológica, la red categorial es un ejercicio de aplicación que puede aplicarse a otras zonas de Bogotá u otras ciudades de similares características que, como se mencionó, pueden ser por su dimensión poblacional. Para la investigación, el siguiente paso es profundizar dentro de la localidad por medio de una revisión histórica de la última mitad del siglo $\mathrm{XX}$, lapso que evidencia de manera contundente la transformación, consolidación y aplicación de las distintas técnicas que surgen de la categorización. Como material de apoyo, se pueden requerir instrumentos como fichas históricas, de levantamiento, ejercicios de categorización con cortes históricos y todos aquellos que forman parte de la percepción.

\section{Bibliografía}

Aguilera, F. (2010). Aplicación de métricas de ecología del paisaje para el análisis de patrones de ocupación urbana en el Área Metropolitana de Granada. Anales de Geografía de la Universidad Complutense, 30 (2), 9-29.

Alexiou, I. (2011). La ciudad del futuro, la ciudad inteligente. Una aproximación sistémica. Bogotá: Pontificia Universidad Javeriana.

Borie, A., y Denieul, F. (1985). Método de análisis morfológico de tejidos urbanos. París: Unesco.

Capel, H. (2002). La morfología de las ciudades. Barcelona: Ediciones El Servial.

Casariego/Guerra, arquitectos S. L. (2008). Plan territorial parcial de ordenación de la comarca de Abona. Santa Cruz de Tenerife: Cabildo Insular de Tenerife.

Castells, M. (1971). Problemas de investigación en sociología urbana. México D. F.: Siglo XXI.

Centro de estudios de la construcción y el desarrollo urbano y regional [Cenac]. (Junio, 2013). 
Boletin estadistico contexo sectorial. Colombia total nacional. Recuperado de www.cenac.org.co

Cuéllar, M., y Mejía, G. (2007). Atlas histórico de Bogotá. Cartografía 1791-2007. Bogotá: Planeta.

Currie, L. (1988). Urbanización y desarrollo, un diseño para el crecimiento metropolitano Bogotá: Camacol, Universidad de los Andes.

De Solá-Morales, M. (1986). Las formas del crecimiento. Lotus International, (51), 19-22.

De Uribina, A., y Zambrano, F. (2009). Impacto de "El Bogotazo" en las actividades residenciales y los servicios de alto rango en el Centro Histórico de Bogotá. Estudio de caso. dearquitectura, (5), 152-165.

Departamento Administrativo Nacional de Estadística [DANE]. (2005). Consulta de información censal. Recuperado de http://190.25.231.242/ cgibin/RpWebEngine.exe/PortalAction? \&MODE $=$ MAIN\&BASE $=$ CG2005ASICO\&MAIN=WebServerMain.inl

Departamento Administrativo Nacional de Estadística [DANE]. (2007). Regional, Informe de coyuntura económica. Recuperado de http://www. dane.gov.co/files/icer/2007/informe_ejec_07.pdf

Gouëset, V. (1998). Bogotá: nacimiento de una metropóli. La originalidad del proceso de concentración urbana en Colombia. Bogotá: Tercer Mundo Editores.

Grupo de investigación en Gestión Urbana. (2008). Gestión urbana, debates y aplicaciones. Bogotá: Universidad Piloto de Colombia.

Janoshka, M. (2006). El modelo de ciudad latinoamericana. Privatización y fragmentación de espacio urbano de Buenos Aires: el caso Nordelta. Recuperado de http://www.michael-janoschka. de/pdfs/Janoschka,\%20Michael\%20(2006)_El_ modelo_de_ciudad_latinoamericana.PDF
Kostof, S. (2009). The City Shaped. Urban Patterns and Meanings Through History. 2a ed. Londres: Thames \& Hudson.

Lynch, K. (2012). La imagen de la ciudad. México: Gustavo Gili.

Nullvalue. (2009, 5 de junio). El siglo XX: población y crecimiento económico. El Tiempo. Recuperado de http://www.eltiempo.com/archivo/ documento/MAM-3473200

Rincón, P. (2007). Bogotá y sus modalidades de ocupación del suelo. Análisis de los procesos de re-densificación. Bogotá: Universidad Nacional de Colombia.

Rodríguez, J., y Villa, M. (1998). Distribución espacial de la población, urbanización y ciudades intermedias: hechos en su contexto. En R. Jordan y D. Siminoni (comps.), Ciudades Intermedias de Latinoamérica y el Caribe. Propuestas para la gestión urbana (pp. 25-32). Roma: MAE.

Rubio, R. (comp.) (2006). Ciudades urgentes. Intervención en áreas urbanas de crecimiento rápido. Bogotá: Universidad de los Andes.

Salazar, J. (2001). ¿Expansión o densificación? Reflexiones en torno al caso Bogotá. Bitácora Urbano/Territorial, (5), 21-35.

Saldías Barreneche, C. (2007). El suelo, una fuente de riqueza colectiva. Cuaderno del informe de desarrollo humano para Bogotá, (4): 55-69.

Secretaría Distrital de Cultura, Recreación y Deportes. (2008). Localidad de Chapinero. Ficha básica. Recuperado de www.culturarecreacionydeporte.gov.co/observatorio/documentos/ localidades/chapinero.pdf

Secretaría Distrital de Movilidad. (2013). Cartografía: OIS-SDM - mapa referencia IDECA. Bogotá: Alcaldía Mayor de Bogotá. 